\title{
SIGNIFICANCE OF COAGULATION FACTORS IN MANAGEMENT OF IDIOPATHIC EPISTAXIS
}

\author{
N. Parusharam Nagula1, G. Madhusudan Reddy², Rajendra Prasad Janga ${ }^{3}$
}

1Associate Professor, Department of ENT, Kakathiya Medical College/ MGM Hospital, Warangal, Telangana. 2 Associate Professor, Department of ENT, Kakathiya Medical College/ MGM Hospital, Warangal, Telangana. ${ }^{3}$ Senior Resident, Department of ENT, Kakathiya Medical College/ MGM Hospital, Warangal, Telangana.

ABSTRACT

\section{BACKGROUND}

Epistaxis or Bleeding from nose is one of the most common condition in ENT Department throughout the world, sometimes necessitating admission in hospital. ${ }^{1}$ While attending the patient for the treatment of Epistaxis to control the bleeding, coagulation screening tests are ordered for in addition to routine blood investigations. But most of the times, epistaxis is due to no identifiable causes known as Idiopathic Epistaxis. In such scenario, the role of coagulation screening tests is unclear. An attempt is made to study whether there is any necessity of asking for coagulation screening tests.

The objective is to study the need of coagulation screening tests in idiopathic epistaxis.

\section{MATERIALS AND METHODS}

The study was performed in patients attending casualty and OPD of Mahatma Gandhi Memorial Hospital, Kakathiya Medical College, Warangal, Telangana. The study was done for two years from August 2014 to July 2016. All the patients who attended casualty or outpatient department or admitted in ENT wards with spontaneous epistaxis were taken into the study. The exclusion criteria were history of trauma, local nasal pathology, systemic disorders like liver and kidney pathology, malignancy, history of bleeding disorders and patients on anticoagulant medications.

All the patients were taken detailed history and examined clinically (ENT and General Examination). Complete blood picture including platelet count and BT, CT and coagulation screening with PT and APTT and INR was done for all the patients who were included in the study. Treatment modalities used to control epistaxis in these patients were electrocautery, anterior nasal packing and posterior nasal packing.

\section{RESULTS}

None of the patients with epistaxis had coagulation profile derangement. Out of 75 patients, 46 (61.33\%) were discharged from Observation (OB) room of casualty after clinical and ENT examination. While $29(38.66 \%)$ patients were admitted in ward for further management, out of these admitted patients 20 (68.96\%) had bilateral Anterior Nasal Packing (ANP) done, 6 patients (20.68\%) needed Posterior Nasal Packing (PNP) and 3 (10.34\%) required Electrocauterisation (EC).

\section{CONCLUSION}

It is found from our study that routine screening for coagulation studies in patients of epistaxis has no role and only it is a financial burden to the patient and hospital. It is recommended from our study only when strong history and proper clinical and ENT examination suggests or indicate, then only coagulation profile screening should be carried out in patients of epistaxis.

\section{KEYWORDS}

Epistaxis, Screening Tests, Coagulation Factors.

HOW TO CITE THIS ARTICLE: Nagula NP, Reddy GM, Janga RP. Significance of coagulation factors in management of idiopathic epistaxis. J. Evolution Med. Dent. Sci. 2018;7(03):338-341, DOI: 10.14260/jemds/2018/75

\section{BACKGROUND}

Epistaxis, bleeding from nose is the commonest condition with which patients attend ENT OPD. Sometimes, it is an emergency requiring admission into the hospital. The causes of Epistaxis may be local or systemic conditions. When there is no identifiable cause, it is also called Idiopathic. Treatment may be local pressure application, anterior nasal packing and posterior nasal packing if necessary. Cauterisation either electric or chemical, embolisation and ligation of the feeding vessel may be the other modalities of treatment depending on

'Financial or Other Competing Interest': None.

Submission 03-11-2017, Peer Review 28-12-2017,

Acceptance 03-01-2018, Published 13-01-2018.

Corresponding Author:

Dr. G. Madhusudan Reddy,

H. No. 2-10-700, Teachers Colony Phase 1,

Waddepally Hanamkonda-District,

Warangal (Urban)-506370.

E-mail:drgmsrent@gmail.com

DOI: $10.14260 /$ jemds $/ 2018 / 75$

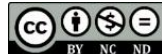

the situation. When dealing with patients of Epistaxis, underlying bleeding and coagulation disorders must be kept in mind which respond well to replacement with blood transfusion, plasma or defective coagulation factors. While attending to such patients with history of bleeding and coagulation disorders, it may be essential to ask for coagulation screening tests in addition to routine tests. But the necessity of asking for coagulation screening tests in patients without such history is unclear. In the opinion of some authors, coagulation screening tests are of little value in the absence of firm indications. ${ }^{2,3}$ But even today, coagulation tests are ordered as a policy of department. ${ }^{4}$

\section{Objective}

To study the need of coagulation screening tests in idiopathic epistaxis.

\section{MATERIALS AND METHODS}

The study was performed in patients attending casualty and OPD of Mahatma Gandhi Memorial Hospital, Kakathiya 
Medical College, Warangal, Telangana. The study was done for two years from August 2014 to July 2016. All the patients who attended casualty or outpatient department or admitted in ENT wards with spontaneous epistaxis were taken into the study. The exclusion criteria were history of trauma, local nasal pathology, systemic disorders like liver and kidney pathology, malignancy, history of bleeding disorders and patients on anticoagulant medications.

All the patients were taken detailed history and examined clinically (ENT and General Examination). Complete blood picture including platelet count and BT, CT and coagulation screening with PT and APTT and INR was done for all the patients who were included in the study. Treatment modalities used to control epistaxis in these patients were electrocautery, anterior nasal packing and posterior nasal packing.

\section{Statistical Methods}

Quantitative or qualitative results will be analysed by simple manual analysis using frequency and percentage.

Study Design- Prospective observational study.

\section{RESULTS}

There were total 75 patients. The age ranging from 8 yrs. to 75 yrs. Out of 75 patients, 52 (69.33) were male and 23 (30.66) were female. Prothrombin Time (PT) of the patients ranged from 11 - 16 seconds with mean being 13.22 seconds. Similarly, activated partial thromboplastin time ranged from 28 - 32 seconds, mean being 28.66 seconds. Bleeding Time (BT) was 2 - 7 minutes (mean 4 - $6 \mathrm{mts}$ ), Clotting Time (CT) ranged between 6 - $10 \mathrm{mts}$ (mean $8.8 \mathrm{mts}$ ) and International Normalised Ratio was between 0.8 and 1.2. As all the patients' reports were within normal limits, we did not further investigate for Factor Analysis. None of the patients with epistaxis had coagulation profile derangement. Out of 75 patients, 46 (61.33\%) were discharged from Observation (OB) room of casualty after clinical and ENT examination. While $29(38.66 \%)$ patients were admitted in ward for further management. Out of these admitted patients 20 (68.96\%) were done bilateral Anterior Nasal Packing (ANP), 6 patients $(20.68 \%)$ needed Posterior Nasal Packing (PNP) and 3 (10.34\%) required Electrocauterisation (EC).

\begin{tabular}{|c|c|c|c|c|c|}
\hline Modalities & Observation & ANP & PNP & EC & Total \\
\hline Number & 46 & 20 & 6 & 3 & 75 \\
\hline \multicolumn{7}{|c|}{ Table 1. Management Modalities } \\
\hline
\end{tabular}

ANP- Anterior Nasal Packing.

PNP- Posterior Nasal Packing.

EC- Electrocauterisation.

\begin{tabular}{|c|c|c|c|c|}
\hline $\begin{array}{c}\text { Coagulation } \\
\text { Tests }\end{array}$ & PT (sec) & $\begin{array}{c}\text { APTT } \\
\text { (sec) }\end{array}$ & CT (min) & BT (min) \\
\hline Mean & 13.22 & 28.66 & 8.8 & 4.6 \\
\hline \multicolumn{5}{|c|}{ Table 2. Mean of Coagulation Profiles } \\
\hline
\end{tabular}

PT- Prothrombin Time.

APTT- Activated Partial Thromboplastin Time (APTT).

CT- Clotting Time.

BT- Bleeding Time.

\begin{tabular}{|c|c|c|}
\hline Coagulation Profile & Normal & Abnormal \\
\hline Number & $75(100 \%)$ & $0(0 \%)$ \\
\hline \multicolumn{2}{|c|}{ Table 3. Results of Coagulation Profile } \\
\hline
\end{tabular}

\section{DISCUSSION}

Epistaxis is the most common emergency condition in otolaryngology. The cause of epistaxis can be local, ${ }^{1}$ systemic or idiopathic. Being emergency patients presenting with epistaxis attend emergency room or casuality where they are mostly seen by medical officers or physicians, most of them ask for routine coagulation screening for all these patients. Identification of cause is important by taking proper history, by general examination and clinical examination as it is very essential in management plan being followed by otolaryngologist or haematologist. 5

\section{Vascular Anatomy}

The nasal blood supply comes from both internal and external carotid artery systems. The external carotid provides arterial flow via the facial and internal maxillary artery (IMA). The facial artery forms the superior labial artery, which supplies the septum and nasal alae. The IMA terminates into 5 branches: the sphenopalatine, pharyngeal, greater palatine, infraorbital and superior alveolar arteries. Of these 5 branches only the sphenopalatine, pharyngeal and greater palatine supply the nasal cavity. The sphenopalatine and its terminal branches supply the septum and middle and inferior turbinate area. The pharyngeal artery also supplies the inferior aspect of the lateral nasal wall, and the greater palatine transverses the greater palatine canal and supplies the anterior aspect of the septum.

The internal carotid artery supplies the nose via the terminal branches of the ophthalmic artery and the anterior and posterior ethmoid arteries. These arteries branch from the ophthalmic artery within the orbit and descend into the nasal cavity from above, supplying the septum and lateral nasal walls. Of note, 2 anastomotic areas within the nose often provide a source of epistaxis. Woodruff area is located on the inferior aspect of the lateral nasal wall, posterior to the inferior turbinate. It is formed from the anastomoses of the sphenopalatine and pharyngeal arteries. The posterior location makes it a common source for severe, non-traumatic bleeds. Kiesselbach plexus is an anastomosis with branches from both the internal and external carotid artery systems. The anterior ethmoid, greater palatine, sphenopalatine and superior labial arteries all form a plexus of vessels in the anteroinferior nasal septum. Kiesselbach plexus is the source of the majority of nose bleeds.

\section{Causes Divided into-}

A. Local, in the nose or nasopharynx.

B. General.

C. Idiopathic.

D. Local Causes.

\section{Nose}

1. Trauma- Finger nail trauma, injuries of nose, intranasal surgery, fractures of middle third of face and base of skull, hard blowing of nose and violent sneeze.

2. Infections- Acute: Viral rhinitis, nasal diphtheria, acute sinusitis. Chronic- All crust-forming diseases, e.g. atrophic rhinitis, rhinitis sicca, tuberculosis, syphilis 
septal perforation, granulomatous lesion of the nose, e.g. rhinosporidiosis.

3. Foreign bodies- Non-Living: Any neglected foreign body, rhinolith.

Living- Maggots, leeches.

4. Neoplasms of nose and paranasal sinuses.

Benign- Haemangioma, papilloma.

Malignant- Carcinoma or sarcoma.

5. Atmospheric changes. High altitudes, sudden decompression (Caisson's disease).

6. Deviated nasal septum.

A. Nasopharynx

1. Adenoiditis.

2. Juvenile angiofibroma.

3. Malignant tumours.

\section{B. General Causes}

1. Cardiovascular system. Hypertension, arteriosclerosis, mitral stenosis, pregnancy (hypertension and hormonal).

2. Disorders of blood and blood vessels. Aplastic anaemia, leukaemia, thrombocytopenic and vascular purpura, haemophilia or Christmas disease, scurvy, vitamin $\mathrm{K}$ deficiency, hereditary haemorrhagic telangiectasia.

3. Liver disease. Hepatic cirrhosis (deficiency of factor II, VII, IX and X).

4. Kidney disease. Chronic nephritis.

5. Drugs. Excessive use of salicylates and other analgesics (as for joint pains or headaches), anticoagulant therapy (for heart disease).

6. Mediastinal compression. Tumours of mediastinum (raised venous pressure in the nose).

7. Acute general infection. Influenza, measles, chickenpox, whooping cough, rheumatic fever, infectious mononucleosis, typhoid, pneumonia, malaria, dengue fever.

\section{Idiopathic}

Many times, the cause of epistaxis is not clear.

\section{Management}

The management of epistaxis can be divided into medical therapy, conservative therapy, surgical therapy and arterial embolisation.

\section{Medical Therapy}

Medical therapy acts in both a therapeutic and prophylactic manner. Hypertension and other haematologic causes for epistaxis must be identified and corrected initially. Once these conditions are corrected, the majority of bleeds are alleviated with gentle direct pressure in the form of a nose pinch. Furthermore, nasal irrigation with saline and the placement of topical ointment in the nostrils create a humidified nasal environment that can prevent further episodes of epistaxis. If bleeding persists, a topical nasal decongestant for the purpose of vasoconstriction can be used. Care must be taken to not continue topical decongestant use indefinitely, as it can become physiologically addictive.

\section{Conservative Therapy}

Conservative therapy includes cautery and nasal packing. Nasal cautery can be performed chemically or thermally.
Chemical cautery uses topical application of silver nitrate, while Bovie electrocautery is used in thermal cautery. Cautery can be performed at the bedside or in the clinical setting after adequate topical anaesthesia if the bleeding site is anterior and therefore visible. More posterior sites may require general anaesthesia and an operating room setting.

Nasal packing is performed via anterior or posterior nasal packs. When medical therapy and cautery fail, identification of anterior nasal bleeds is followed by application of an anterior pack. Physician preference and the patient's comfort level determine the choice of packing agent. All packs should be covered in antibiotic ointment and provide ample pressure against the source of bleeding. Packs should be left in place no more than 5 days to prevent toxic shock syndrome or other associated infections. Oral antibiotics should be administered for as long as the packs remain in the nose. After placement of an anterior pack, epistaxis should cease, and examination should reveal no active bleeding down the posterior oropharynx. After successful placement of an anterior pack, patients can be sent home and safely managed on an outpatient basis.

If epistaxis begins in the contralateral nose or bleeding down the posterior oropharynx worsens after application of an anterior pack, the addition of a posterior pack may be required. Posterior packs include double-balloon nasal packs as well as Foley catheters. The goal of the posterior pack is to seal the nasopharynx at the choanal entrance and provide a support against which to place an anterior pack. Doubleballoon systems provide both an anterior and posterior balloon. When using a Foley catheter, the placement of anterior packing material tightly against the inflated Foley balloon is required. Similar general principles must be followed when using posterior packs to prevent infection. One important difference is that the placement of an anterior/ posterior pack requires hospitalisation secondary to potential complications. Specifically, placing a pack in the nasopharynx can trigger the nasopulmonary reflex, resulting in apnoea and dysrhythmias. Therefore, patients should be monitored in an intensive care unit or with continuous pulse oximetry and telemetry.

\section{Surgical Therapy}

Surgical therapy for epistaxis has largely been supplanted by the use of arterial embolisation. Procedures used for bleeds that are refractory to medical and conservative therapy include IMA, anterior ethmoid artery and external carotid artery ligation.

\section{Arterial Embolisation}

Arterial embolisation performed by interventional radiologists is a relatively new technique used to embolise distal branches of the IMA. Under local anaesthesia, diagnostic angiograms are performed to assess the vascular anatomy. Brisk bleeds will show up as blushes and can be selectively embolised. Potential complications include transient hemiparesis, facial paralysis, blindness, columellar necrosis, stroke and death, but these are uncommon when procedures are performed by experienced surgeons. Finally, a general principle is that the more proximal the embolisation, the greater the likelihood for post embolisation complications. 
In addition to managing acute bleeding symptoms, emergency health care providers should be aware of the clinical indications that a bleeding disorder may be present. Coagulation disorders should be considered in children with a history of recurrent, prolonged, severe or refractory bleeding and for individuals with a history of easy bruising, other severe bleeding or gingival bleeding. The presence of petechiae may be a particularly visible indication of additional bleeding symptoms. Previous inpatient admissions or emergency care for bleeding symptoms, the presence of iron deficiency anaemia and a family history of excessive bleeding may also be useful in identifying those at an increased risk for bleeding disorders. Suspected cases of platelet disorders should be referred to a haematologist even if initial haematologic assessments including blood counts and PT/PTT appear normal.

PT measures extrinsic and APTT measures intrinsic pathway of coagulation process. Coagulation factor deficiency is less common, so the specific factor analysis is not required usually but can be done when indicated by history and examination are suggestive of the same. The chances of finding abnormal coagulation reports in an otherwise normal people without any co-morbidity is as low as $1 \% .6,7$ The role of routine coagulation screening in management of epistaxis is not clear. In our study, we could not find a single patient with epistaxis who had abnormal coagulation report. Hence, our study supports few authors' opinion of indiscriminate request for coagulation studies are not beneficial to the patient and moreover they are unnecessary expenditure to the patient and hospital.8,9

Management of epistaxis in any age group essentially assessing the patient, establishing the site of bleeding and stopping the bleeding by various means. Epistaxis can be minimal or sometimes troubling and life-threatening also. In our study, most of the patients are discharged from observation room only. Few patients were admitted for further treatment, either by anterior nasal packing or posterior nasal packing or by electrocautery. None of our patients needed ligation of major vessels.

\section{CONCLUSION}

It is found from our study that routine screening for coagulation studies in patients of epistaxis has no role and only it is a financial burden to the patient and hospital.

It is recommended from our study only when strong history and proper clinical and ENT examination suggest or indicate, then only coagulation profile screening should be carried out in patients of epistaxis.

\section{REFERENCES}

[1] Douglas R, Wormald PJ. Update on epistaxis. Curr Opin Otolaryngol Head Neck Surg 2007;15(3):180-3.

[2] Pande NM, Jain SKT, Mahore DM, et al. Role of routine coagulation studies in idiopathic epistaxis. Indian Journal of Otolaryngology and Head and Neck Surgery 2006;58(2):160-1.

[3] Thaha MA, Nilssen EL, Holland S, et al. Routine coagulation screening in the management of emergency admission for epistaxis-is it necessary? J Laryngol Otol 2000;114(1):38-40.

[4] Holland S, Thaha MA, Nilssen EL, et al. Coagulation studies in patients admitted with epistaxis-current practice in Scotland. J Laryngol Otol 1999;113(12): 1086-8.

[5] Wahab MSA, Fathy H, Ismail R, et al. Recurrent epistaxis in children: when should we suspect coagulopathy? The Egyptian Journal of Otolaryngology 2014;30(2):106-11.

[6] Pollice PA, Yoder MG. Epistaxis: a retrospective review of hospitalized patients. Otolaryngol Head Neck Surg 1997;117(1):49-53.

[7] Auble TE, Taylor DM, Hsu EB, et al. Evaluation of guidelines for ordering prothrombin and partial thromboplastin times. Acad Emerg Med 2002;9(6):567-74.

[8] Jackson KR, Jackson RT. Factors associated with active, refractory epistaxis. Arch Otolaryngol Head Neck Surg 1988;114(8):862-5.

[9] Smith IM, Ludlam CA, Murray JA. Haematological indices in elderly patients with epistaxis. Health Bull (Edinb) 1988;46(5):277-81. 